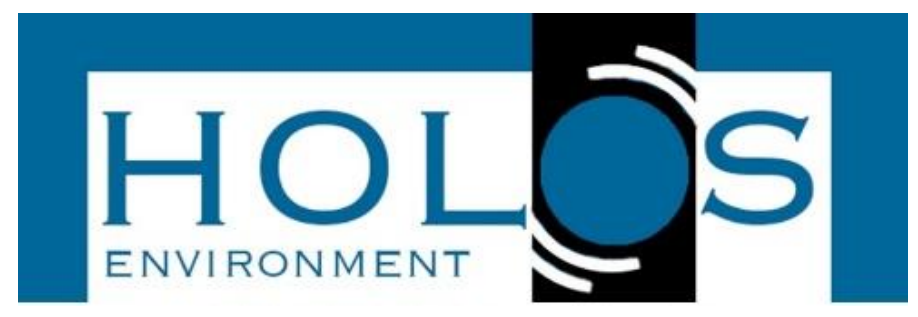

\title{
ANÁLISE DAS ALTERAÇÕES DOS PARÂMETROS DE QUALIDADE DA ÁGUA DO RIO DO CARMO, AFLUENTE DO RIO DOCE, APÓS ROMPIMENTO DA BARRAGEM DE FUNDÃO, EM MARIANA-MG
}

\section{ANALYSIS OF THE CHANGES ON THE WATER QUALITY PARAMETERS OF THE CARMO RIVER, DOCE RIVER TRIBUTARY, AFTER THE FUNDÃO DAM BREAK, IN MARIANA-MG}

\author{
Alexandre Sylvio Vieira da Costa ${ }^{1}$; Clara Diniz Oliveira Vasconcellos ${ }^{1}$; \\ Tamila Caliman Bravin ${ }^{1}$
}

Artigo recebido em: 03/04/2018 e aceito para publicação em: 06/09/2018.

DOI: http://dx.doi.org/10.14295/holos.v18i2.12280

Resumo: A água é o principal recurso para vida no planeta, estando relacionada à sobrevivência das espécies e ao equilíbrio da biodiversidade. A poluição aquática é consequência principalmente das atividades humanas. No dia 5 de novembro de 2015, a barragem de rejeitos de Fundão que armazenava os rejeitos da mineração de ferro da empresa Samarco se rompeu, atingindo o rio Gualaxo do Norte, desaguando no rio do Carmo até a sua chegada no rio Doce. O estudo objetivou avaliar a qualidade das águas do rio do Carmo por meio dos dados disponibilizados pelo IGAM (Instituto de Gestão de Águas de Minas Gerais) em dois pontos de monitoramento, RD009 e RD071, comparando os resultados com os limites permitidos pela legislação vigente e realizar análise relacionando os resultados observados com o rompimento da barragem de Fundão em Mariana-MG. Foi selecionado o período de 2013 a 2017 e após filtragem dos dados, foram analisados os parâmetros inorgânicos alumínio dissolvido, arsênio total, bário total, cádmio total, chumbo total, cianeto livre, cloreto total, cobre dissolvido, cromo total, ferro dissolvido, fósforo total, manganês total, mercúrio total, níquel total, nitrato, nitrito, nitrogênio amoniacal total, sulfato total, sulfeto e zinco total e demais parâmetros como clorofila "a", sólidos dissolvidos totais, fenóis totais e cor verdadeira, demanda bioquímica de oxigênio (DBO), óleos e graxas, oxigênio dissolvido, turbidez e pH in loco. No geral os parâmetros atenderam a legislação vigente, com exceção do fósforo, manganês, alumínio e arsênio. Apenas o parâmetro turbidez demonstrou possível relação com o rompimento da barragem.

Palavras-chave: Rompimento Barragem. Qualidade da Água. Poluição Hídrica.

Abstract: Water is the main resource for life on the planet, being related to the survival of species and the balance of biodiversity. Water pollution is a consequence of human activities. On November 5, 2015, the Fundão dam break, reaching the Gualaxo do Norte river, flowing into the Carmo river until its arrival in the Doce river. The objective of this study was to evaluate the quality of the waters of the Carmo River by means of the data provided by IGAM (Water Management Institute of Minas Gerais) at two monitoring points, RD009 and RD071, comparing the results with the limits allowed by the current legislation and performing analysis relating the results observed with the disruption of the Fundão dam in Mariana-MG. The following parameters were selected: Dissolved aluminum, total arsenic, total barium, total cadmium, total lead, free cyanide, total chloride, dissolved copper, total chromium, dissolved iron, phosphorus total sulfur, sulfide and total zinc and

\footnotetext{
1 Universidade Federal dos Vales do Jequitinhonha e Mucuri (UFVJM), Teófilo Otoni, MG. Email:

(asylvio@hotmail.com, clarinhhadiniz@hotmail.com,tamila.ambiente@gmail.com)
} 
other parameters such as chlorophyll "a", total dissolved solids, total phenols and surface active substances, true color, biochemical demand, total alkalinity, total manganese, total mercury, total nickel, nitrate, nitrite, total ammoniacal nitrogen, of oxygen (BOD), oils and greases, dissolved oxygen, turbidity and $\mathrm{pH}$. In general, the parameters met the current legislation with the exception of phosphorus, manganese, aluminum and arsenic. Only the parameter turbidity demonstrated a possible relation with the dam rupture.

Keywords: Dam Break. Water Quality. Water-Poluttion.

\section{INTRODUÇÃO}

O crescimento demográfico promove desequilíbrios ambientais. A exploração e o consumo desordenado dos recursos naturais apresentam um sério risco a sobrevivência da humanidade e do próprio planeta (CAVACO, 1992).

A água é o principal recurso relacionado à sobrevivência das espécies e o equilíbrio da biodiversidade. Grande parte da superfície terrestre é coberta por água dos oceanos, rios e lagos, além da presença da água em aquíferos e geleiras, totalizando aproximadamente $1.370 .000 .000 \mathrm{~km}^{3}$. Apesar deste extenso volume, $97 \%$ do total é constituído por água salgada, impossibilitando o consumo direto (VICTORINO, 2007).

Apesar de sua importância, o ser humano, de modo geral, utiliza a água doce de forma indiscriminada, esgotando as reservas existentes. A poluição derivada da atividade antrópica também tem comprometido os mananciais hídricos. Dentre as diversas formas de agressão aos recursos hídricos, são identificadas a poluição térmica, sedimentar, biológica, radioativa e química (ROSA et al., 2012).

As atividades industriais são responsáveis por gerar intensa poluição quando operam sem padrão de controle dos seus sistemas produtivos ou com proporcionalidades muito dispares entre a poluição e sua mitigação. Considerando a fonte, poluentes como os metais podem ser introduzidos no ecossistema aquático por diferentes atividades como mineração, galvanoplastia, curtumes e manufaturas de produtos eletrônicos (LIMA e MERÇON, 2011; PEREIRA, 2004).

A Lei 9.433/1997 que instituiu a Política Nacional de Recursos Hídricos, e as demais leis federais e estaduais relacionadas, dispõem sobre a gestão das águas planejamento e controle, estabelecendo regras para a proteção dos recursos hídricos. A supracitada legislação define que a gestão dos recursos hídricos deve sempre proporcionar o uso múltiplo das águas e que em situações de escassez, o uso prioritário dos recursos hídricos é o consumo humano e a dessedentação de animais (PRNH, 1997). 
Entre as variáveis de importância para a poluição das águas superficiais, pode-se citar a localização do corpo hídrico, o estado da preservação da mata ciliar e também as atividades econômicas desenvolvidas na bacia hidrográfica onde o corpo hídrico se localiza. Os metais, por exemplo, são introduzidos nos sistemas aquáticos por meio da contribuição antrópica ou por fontes naturais através de processos geoquímicos, no intemperismo (YABE; OLIVEIRA, 1998).

Desta forma, o monitoramento dos rios localizados em bacias com grande desenvolvimento de atividades industriais e agrícolas é de extrema importância, de forma a gerar conhecimento que permita uma melhor gestão do meio ambiente e da saúde pública (MACHADO et al., 2016).

Neste contexto, através do projeto Águas de Minas, o Instituto Mineiro de Gestão das Águas (IGAM) realiza o monitoramento das águas superficiais no território mineiro desde o ano de 1997. Este monitoramento contempla 64 estações de amostragem de água, onde são realizadas coletas e análises laboratoriais com periodicidade trimestral de aproximadamente 50 parâmetros físico, químicos e hidrobiológicos. Os principais objetivos do monitoramento realizado pelo IGAM são conhecer e avaliar as condições da qualidade das águas superficiais em Minas Gerais, divulgar a situação de qualidade das águas para os usuários e apoiar o estabelecimento de metas de qualidade, fornecer subsídios para o planejamento da gestão dos recursos hídricos, verificar a efetividade de ações de controle ambiental implementadas e propor prioridades de atuação (IGAM, 2016).

No dia 5 de novembro de 2015, a barragem do Fundão que armazenava os rejeitos do tratamento de minério de ferro da empresa SAMARCO se rompeu. Na catástrofe, 17 pessoas morreram, dois desapareceram e cerca de 1600 ha nas margens do Rio Doce e dos seus afluentes foram recobertos por um rejeito carregado de substâncias químicas do tratamento do minério de ferro. A população das mais de 200 cidades e vilarejos da bacia do Rio Doce foram afetadas. A localidade de Bento Rodrigues foi a primeira a ser atingida pela onda de rejeitos, que em seguida, atingiu o rio Gualaxo do Norte, desaguando no rio do Carmo até a sua chegada no rio Doce.

O rompimento da barragem de Fundão em Mariana-MG atingiu diretamente os Estados de Minas Gerais e Espírito Santo. Há indícios de que no deságue no mar, chegou a contaminar áreas pertencentes ao estado da Bahia. $O$ acidente provocou grandes impactos ambientais, sociais e econômicos. 
Os prejuízos socioambientais levaram o Governo de Minas a decretar "situação de emergência" para 32 municípios da bacia do rio Doce, sendo mais de 4000 pessoas atingidas diretamente pelo rompimento da barragem de Fundão. Foram impactados diretamente pelos rejeitos municípios e vilarejos; assoreados rios, lagos e córregos causando ampla mortandade de fauna e flora aquática (MINAS GERAIS, 2016).

Desta forma, considerando o contexto das pressões antrópicas e o desastre ocorrido na bacia do rio Doce, este artigo objetivou avaliar a qualidade das águas do rio do Carmo em dois pontos de monitoramento, comparando-se os resultados com os limites permitidos pela legislação vigente e realizar análise relacionando os resultados observados com o rompimento da barragem de Fundão em Mariana-MG.

\section{METODOLOGIA}

\section{1 Área de estudo:}

O rio Doce é formado a partir da confluência do rio Piranga e rio do Carmo, entre as cidades de Ponte Nova, Rio Doce e Santa Cruz do Escalvado, em Minas Gerais no Sudeste do Brasil. A área de drenagem da bacia do rio Doce corresponde a aproximadamente de 84 mil km² , onde $86 \%$ estão em Minas Gerais e 14\% no Espírito Santo. Seu curso é de 886 $\mathrm{km}$ desde a nascente do rio Xopotó até a sua foz no Oceano Atlântico localizada no município de Linhares, no Espírito Santo. São 225 municípios que fazem parte da bacia hidrográfica, 200 do estado de Minas e 25 capixabas (ANA 2016).

O rio do Carmo, objeto de estudo, se origina no município de Ouro Preto, e tem como principais afluentes os rios Gualaxo do Sul e Gualaxo do Norte, este o primeiro rio a ser impactado pelo rejeito do rompimento da barragem de Fundão em Mariana-MG. A bacia hidrográfica do rio do Carmo ocupa uma área de aproximadamente $2.278 \mathrm{~km}^{2}$ e está localizada na Unidade de Planejamento e Gestão dos Recursos Hídricos (UPGRH) DO1 Bacia Hidrográfica do rio Piranga (IGAM, 2010).

O rio do Carmo percorre cerca de $134 \mathrm{~km}$. É formado pelo ribeirão do Funil e o córrego do Tripuí, que nascem na Serrado Veloso, a sua foz encontra-se no município de Rio Doce, na confluência com o rio Piranga, formando o rio Doce (BARBOSA, 2005). As 
minas de Alegria da Samarco Mineração S/A localizam-se nas cabeceiras do rio do Carmo, em território dos municípios mineiros de Ouro Preto e Mariana.

O rio do Carmo não possui enquadramento estabelecido (IGAM, 2010), desta forma, conforme preconizado pela Resolução do Conselho Nacional do Meio Ambiente ํo 357/2005 (CONAMA, 2005), em seu artigo 42, enquanto não aprovados os respectivos enquadramentos, as águas doces serão consideradas como Classe 2, exceto se as condições de qualidade atuais forem melhores, o que determinará a aplicação da classe mais rigorosa correspondente.

Para este estudo, dois pontos de monitoramento foram selecionados (Figura 01). Dentre os dados disponibilizados pelo IGAM, utilizamos os pontos RD009 e RD071, sendo estes localizados à montante e à jusante, respectivamente, do ponto em que o rio Gualaxo do Norte, deságua no rio do Carmo. Tais pontos foram escolhidos de maneira a avaliar o contexto de qualidade da água do rio do Carmo nos últimos anos bem como poder avaliar o impacto causado sobre o curso d'água após o rompimento da barragem de fundão. $O$ ponto de coleta RD009 não foi atingido pelos rejeitos do rompimento da barragem enquanto o ponto RD071 sofreu interferência direta do movimento dos rejeitos.

Figura 01- Pontos de Monitoramento avaliados

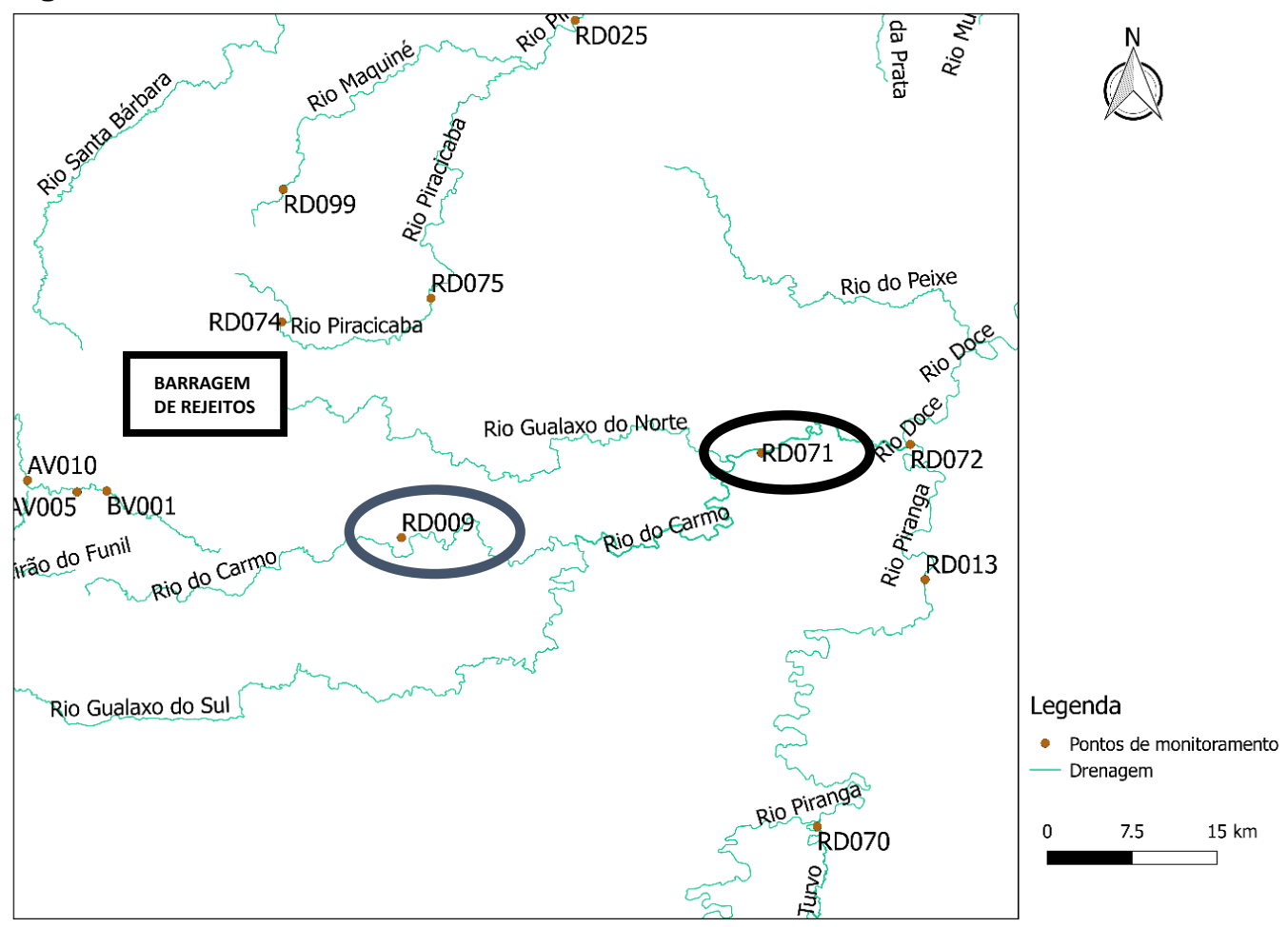

Fonte: Dados digitais: IGAM (2017). Elaboração do autor 


\subsection{Tratamento e análise dos dados:}

No Estado de Minas Gerais, o monitoramento das águas é realizado pelo Instituto Mineiro de Gestão das Águas - IGAM, por meio do Projeto Águas de Minas, em execução desde 1997.

Foram coletados os dados de qualidade da água disponibilizados pelo IGAM em sua plataforma digital Portal InfoHidro para o rio do Carmo do período de janeiro de 2013 a janeiro de 2017 para os pontos de monitoramento de interesse, RD009 e RD071.

Foram selecionados para este trabalho os parâmetros que contém limites estabelecidos pelas legislações federal e estadual vigentes, de maneira a permitir a avaliação dos resultados da análise para os parâmetros no contexto dos limites legais.

Desta forma, após filtragem dos dados, os mesmos foram tabelados e os resultados foram analisados para os seguintes parâmetros:

- Parâmetros inorgânicos: Alumínio dissolvido (mg/L), arsênio total ( $\mathrm{mg} / \mathrm{L})$, bário total (mg/L), cádmio total ( $\mathrm{mg} / \mathrm{L})$, chumbo total $(\mathrm{mg} / \mathrm{L})$, cianeto livre $(\mathrm{mg} / \mathrm{L})$, cloreto total $(\mathrm{mg} / \mathrm{L})$, cobre dissolvido $(\mathrm{mg} / \mathrm{L})$, cromo total, ferro dissolvido $(\mathrm{mg} / \mathrm{L})$, fósforo total (mg/L), manganês total $(\mathrm{mg} / \mathrm{L})$, mercúrio total $(\mathrm{mg} / \mathrm{L})$, níquel total $(\mathrm{mg} / \mathrm{L})$, nitrato $(\mathrm{mg} / \mathrm{L})$, nitrito $(\mathrm{mg} / \mathrm{L})$, nitrogênio amoniacal total $(\mathrm{mg} / \mathrm{L})$, sulfato total $(\mathrm{mg} / \mathrm{L})$, sulfeto e zinco total $(\mathrm{mg} / \mathrm{L})$;

- Demais parâmetros: Clorofila "a" (ug/L), sólidos dissolvidos totais (mg/L), fenóis totais $(\mathrm{mg} / \mathrm{L})$, cor verdadeira, demanda bioquímica de oxigênio (DBO) $\left(\mathrm{mgO}_{2} / \mathrm{L}\right)$, óleos e graxas (mg/L), oxigênio dissolvido (mg/L), turbidez (UNT) e pH in loco.

Os resultados obtidos foram comparados com os limites estabelecidos pela Resolução CONAMA no 357/2005 (BRASIL, 2005) e suas alterações que tratam da classificação dos corpos hídricos de acordo com seus usos pretendidos; e pela Deliberação Normativa Conjunta COPAM/CERH-MG no 01, de 05 de maio de 2008, que dispõe sobre a classificação dos corpos de água e diretrizes ambientais para o seu enquadramento bem como estabelece as condições e padrões de lançamento de efluentes, e dá outras providências. 
Além da verificação com o atendimento da legislação vigente, os dados foram comparados para verificar se houve alteração dos parâmetros confrontando-se os dados a montante e à jusante do ponto de desague do rio Gualaxo do Norte no rio do Carmo, ponto a partir do qual, o rio do Carmo passou a receber os rejeitos da barragem rompida de Fundão.

\section{RESULTADOS E DISCUSSÃO}

\subsection{Análise dos parâmetros inorgânicos}

O deslocamento dos rejeitos da empresa de mineração causou grandes estragos no rio Gualaxo do Norte, o primeiro a receber o material, seguido pelo rio do Carmo e, finalmente, alcançando o rio Doce. Avaliamos a qualidade das águas antes do impacto dos rejeitos no ponto RD071, a partir de pouco mais de dois meses após o rompimento da barragem de rejeitos. O ponto do rio do Carmo RD009, também avaliado, não foi atingido pelos rejeitos.

$\mathrm{Na}$ Tabela 1, observamos a variação da concentração dos elementos alumínio, arsênio, bário, cadmio e chumbo nos dois pontos avaliados do rio do Carmo. Em relação ao alumínio, observa-se que, antes do rompimento da barragem entre janeiro de $2013 \mathrm{e}$ outubro de 2015, foi identificada apenas uma leitura acima do limite preconizado pela CONAMA, de um total de seis leituras, no ponto RD009. O ponto RD071, na rota dos rejeitos, apresentou três leituras de alumínio fora das conformidades da resolução, de um total de 12 leituras. Após o rompimento da barragem da mineradora, verifica-se que entre 11/01/2016 e 11/01/2017, foram realizadas oito leituras no ponto RD071, local da passagem dos rejeitos, com valores acima dos limites em apenas uma leitura. Estes resultados indicam que os efeitos da passagem do alumínio presente nos rejeitos foram de reduzida duração. Antes do período de dois meses após o rompimento, os valores na água do rio já haviam normalizado.

Em relação ao Arsênio, um metal pesado de elevada toxidade, presente na composição mineralógica da região e nos rejeitos da barragem, verificamos que, no ponto RD009, fora da rota dos rejeitos e antes do rompimento da barragem, de um total de 12 amostras coletadas entre 15/01/2013 e 06/10/2015, dez delas apresentavam valores acima 
do preconizado pela Resolução CONAMA 357. No ponto RD071, antes do rompimento, de 12 amostras de água coletadas, apenas duas apresentaram valores fora dos limites. Nas amostras de água coletadas após o rompimento da barragem, no ponto RD009 (sem passagem do rejeito), onde foram coletadas cinco amostras, todas apresentaram valores acima do limite CONAMA. No ponto RD071, onde ocorreu a passagem dos rejeitos, de oito amostras de água coletada, nenhuma apresentou valores acima do preconizado na resolução. Estes resultados demonstram que, apesar da constatação da presença de arsênio nos rejeitos da barragem que rompeu, após algumas semanas, uma parte deste metal foi carreado a jusante, não sendo identificado metal residual na água, ou que ainda esteja ocorrendo escape na barragem. Parte deste metal liberado da barragem provavelmente também está depositado no leito do rio do Carmo, mas mesmo com a coleta de água no período chuvoso, onde ocorre movimento de material do leito do rio, não foi detectado o metal em níveis acima dos preconizados pela resolução CONAMA. Em relação ao ponto de análise onde o rejeito da mineração não atingiu (RD009), os problemas com arsênio são graves, identificados antes e depois do rompimento. As atenções na região estão voltadas exclusivamente para a barragem rompida e o percurso do rejeito, mas o processo de agressão ambiental continua, de forma intensa, em outros pontos da região e a presença de arsênio nas águas é um indicativo.

A exposição ao arsênio provoca diferente manifestações clínicas dependendo da espécie química de arsênio envolvida, bem como a dose e duração da exposição. Em longo prazo, a exposição oral é capaz de conduzir a manifestações sistêmicas em vários órgãos, que são classificadas como carcinogênicas ou não-carcinogênicas (RHODES,2010). Rosa et al. (2012) abordam efeitos da intoxicação humana por arsênio incluem conjuntivite, hiperqueratose, hiperpigmentação, doenças cardiovasculares, distúrbios no sistema nervoso central e vascular periférico, gangrena nos membros e risco de câncer.

Em relação ao chumbo total, há registro de apenas uma data, no ano de 2013, anterior ao acidente do rompimento, de não conformidade com as legislações em análise, não sendo possível afirmar através destes dados uma relação com o acidente. $A$ intoxicação por chumbo é mais perceptível em recém nascidos, crianças de até 6 anos de idade, feto e mulheres gravidas. Esse metal possui características de se bioacumular e dentre as consequências a exposição os efeitos sobre o sistema nervoso central podem 
ser graves bem como causar anemia (WHO, 2011). Em relação ao cadmio, não foi observada qualquer alteração nos seus valores em relação a resolução CONAMA.

Tabela 01- Parâmetros inorgânicos (alumínio dissolvido, arsênio, bário, cadmio e chumbo totais, mg/L) antes do rompimento da barragem em Mariana (preto) e após o rompimento (vermelho) em pontos do Rio do Carmo sem interferência dos rejeitos da mineração (RD009) e com interferência (RD071)

\begin{tabular}{|c|c|c|c|c|c|c|c|c|c|c|}
\hline & \multicolumn{2}{|c|}{$\begin{array}{l}\text { Alumínio } \\
\text { dissolvido }\end{array}$} & \multicolumn{2}{|c|}{ Arsênio Total } & \multicolumn{2}{|c|}{ Bário Total } & \multicolumn{2}{|c|}{ Cádmio Total } & \multicolumn{2}{|c|}{ Chumbo Total } \\
\hline Data & RD009 & RD071 & RD009 & RD071 & RD009 & RD071 & RD009 & RD071 & RD009 & RD071 \\
\hline $15 / 01 / 2013$ & 0,100 & 0,105 & 0,0176 & 0,0144 & 0,0876 & 0,0899 & 0,0005 & 0,0005 & 0,005 & 0,005 \\
\hline $09 / 04 / 2013$ & - & 0,209 & 0,0441 & 0,0228 & - & - & - & 0,0005 & - & 0,014 \\
\hline $09 / 07 / 2013$ & 0,100 & 0,100 & 0,0132 & 0,0003 & 0,0432 & 0,0181 & 0,0005 & 0,0005 & 0,005 & 0,005 \\
\hline $01 / 10 / 2013$ & - & 0,100 & 0,0199 & 0,0050 & - & - & - & 0,0005 & - & 0,005 \\
\hline $14 / 01 / 2014$ & 0,100 & 0,100 & 0,0143 & 0,0055 & 0,0496 & 0,0285 & 0,0005 & 0,0005 & 0,005 & 0,005 \\
\hline $01 / 04 / 2014$ & - & 0,100 & 0,0140 & 0,0021 & - & - & - & 0,0005 & - & 0,005 \\
\hline $08 / 07 / 2014$ & 0,100 & 0,100 & 0,0026 & 0,0023 & 0,0691 & 0,0199 & 0,0005 & 0,0005 & 0,005 & 0,005 \\
\hline 01/10/2014 & - & 0,100 & 0,0010 & 0,0032 & - & - & - & 0,0005 & - & 0,005 \\
\hline $13 / 01 / 2015$ & 0,106 & 0,100 & 0,0202 & 0,0030 & 0,0530 & 0,0173 & 0,0005 & 0,0005 & 0,005 & 0,005 \\
\hline $07 / 04 / 2015$ & - & 0,105 & 0,0153 & 0,0046 & - & - & - & 0,0005 & - & 0,005 \\
\hline $07 / 07 / 2015$ & 0,100 & 0,100 & 0,0192 & 0,0022 & 0,0410 & 0,0200 & 0,0005 & 0,0005 & 0,005 & 0,005 \\
\hline $06 / 10 / 2015$ & & 0,100 & 0,0249 & 0,0029 & - & - & - & 0,0005 & - & 0,005 \\
\hline $11 / 01 / 2016$ & - & - & - & - & - & - & - & - & - & - \\
\hline $12 / 01 / 2016$ & 0,100 & 0,100 & 0,0184 & 0,0010 & 0,0733 & 0,0776 & 0,0005 & 0,0005 & 0,005 & 0,009 \\
\hline $05 / 04 / 2016$ & - & 0,100 & 0,0102 & 0,0018 & - & - & - & 0,0005 & & 0,005 \\
\hline $05 / 07 / 2016$ & 0,100 & 0,100 & 0,0189 & 0,0015 & 0,0460 & 0,0208 & 0,0005 & 0,0005 & 0,005 & 0,005 \\
\hline $30 / 09 / 2016$ & - & 0,100 & - & 0,0034 & - & - & - & 0,0005 & - & 0,005 \\
\hline $18 / 10 / 2016$ & - & 0,100 & 0,0220 & 0,0031 & - & - & - & 0,0005 & - & 0,005 \\
\hline $07 / 11 / 2016$ & - & 0,100 & - & 0,0032 & - & - & - & 0,0005 & - & 0,005 \\
\hline $05 / 12 / 2016$ & - & 0,133 & - & 0,0032 & - & - & - & 0,0005 & - & 0,007 \\
\hline $10 / 01 / 2017$ & 0,100 & 0,100 & 0,0139 & 0,0024 & 0,0490 & 0,0250 & 0,0005 & 0,0005 & 0,005 & 0,005 \\
\hline LIMITES & \multicolumn{2}{|c|}{0,100} & \multicolumn{2}{|c|}{0,0100} & \multicolumn{2}{|c|}{0,7000} & \multicolumn{2}{|c|}{0,0010} & \multicolumn{2}{|c|}{0,010} \\
\hline
\end{tabular}

Fonte: Dados: IGAM (2017). Elaboração do autor. Valores acima dos limites estabelecidos pela Resolução CONAMA nº 357/2005 (negrito)

Na Tabela 2 foram dispostos os valores de Cianeto livre, cloreto total, cobre, cromo e ferro dissolvido. Nas dezenas de análises realizadas verifica-se que não ocorreu nenhuma leitura destes elementos com valores acima do preconizado pela resolução CONAMA 357. Estes resultados corroboram com as análises químicas realizadas nos rejeitos que indicam valores reduzidos destes elementos, com exceção do ferro. Este último elemento foi identificado em quantidades mais elevadas nos rejeitos, mas podemos verificar, que poucas semanas após o rompimento da barragem, não registrou-se qualquer sequela na água do rio do Carmo no ponto de passagem do rejeito. Podemos deduzir que ocorreu um intenso carreamento deste elemento para jusante e que uma parte deste elemento provavelmente está depositado no leito do rio, considerando o elevado grau de assoreamento dos rios da região após a passagem do rejeito. 
Tabela 02- Parâmetros inorgânicos (Cianeto livre, cloreto total, cobre dissolvido, cromo total e ferro dissolvido, $\mathrm{mg} / \mathrm{L}$ ) antes do rompimento da barragem em Mariana (preto) e após o rompimento (vermelho) em pontos do Rio do Carmo sem interferência dos rejeitos da mineração (RD009) e com interferência (RD071)

\begin{tabular}{|c|c|c|c|c|c|c|c|c|c|c|}
\hline \multirow{3}{*}{ Data } & \multicolumn{2}{|c|}{ Cianeto Livre } & \multicolumn{2}{|c|}{ Cloreto Total } & \multicolumn{2}{|c|}{ Cobre Dissolvido } & \multicolumn{2}{|c|}{ Cromo Total } & \multicolumn{2}{|c|}{ Ferro Dissolvido } \\
\hline & RDnח9 & RDO71 & BDnng & RD007 & RDnng & RDOח71 & & & & RD007 \\
\hline & & & & 1 & & RDOU/ & RD009 & RD0071 & RD009 & 1 \\
\hline 15/01/13 & 0,002 & 0,002 & 3,11 & 2,24 & 0,004 & 0,004 & 0,04 & 0,04 & 0,141 & 0,251 \\
\hline $09 / 04 / 13$ & - & 0,002 & 1,86 & 2,15 & 0,004 & 0,004 & - & 0,04 & - & 0,254 \\
\hline 09/07/13 & 0,002 & 0,002 & 3,3 & 1,49 & 0,004 & 0,004 & 0,04 & 0,04 & 0,110 & 0,100 \\
\hline $01 / 10 / 13$ & - & 0,002 & 4,68 & 2,77 & 0,004 & 0,004 & - & 0,04 & - & 0,167 \\
\hline $14 / 01 / 14$ & 0,002 & 0,003 & 3,27 & 1,48 & 0,004 & 0,008 & 0,04 & 0,04 & 0,056 & 0,070 \\
\hline $01 / 04 / 14$ & - & 0,002 & 3,25 & 1,66 & 0,004 & 0,004 & - & 0,04 & - & 0,049 \\
\hline 08/07/14 & 0,002 & 0,002 & 2,87 & 1,77 & 0,004 & 0,004 & 0,04 & 0,04 & 0,161 & 0,111 \\
\hline $01 / 10 / 14$ & - & 0,002 & 7,41 & 2,92 & 0,004 & 0,004 & - & 0,04 & - & 0,090 \\
\hline $13 / 01 / 15$ & 0,002 & 0,002 & 4,27 & 1,86 & 0,004 & 0,004 & 0,04 & 0,04 & 0,158 & 0,174 \\
\hline $07 / 04 / 15$ & - & 0,003 & 3,25 & 1,94 & 0,004 & 0,004 & - & 0,04 & - & 0,279 \\
\hline $07 / 07 / 15$ & 0,002 & 0,002 & 4,33 & 1,75 & 0,004 & 0,004 & 0,04 & 0,04 & 0,084 & 0,054 \\
\hline $06 / 10 / 15$ & - & 0,002 & 4,82 & 1,88 & 0,004 & 0,004 & - & 0,04 & - & 0,097 \\
\hline $11 / 01 / 16$ & - & - & - & - & - & - & - & - & - & - \\
\hline $12 / 01 / 16$ & 0,003 & 0,002 & 4,57 & 1,9 & 0,004 & 0,004 & 0,04 & 0,04 & 0,185 & 0,069 \\
\hline $05 / 04 / 16$ & & 0,002 & 4,37 & 1,73 & - & - & - & 0,04 & - & 0,204 \\
\hline $05 / 07 / 16$ & 0,002 & 0,002 & 4,94 & 1,6 & 0,004 & 0,004 & 0,04 & 0,04 & 0,136 & 0,148 \\
\hline $30 / 09 / 16$ & - & - & - & 1,6 & - & 0,004 & - & 0,04 & - & 0,160 \\
\hline $18 / 10 / 16$ & - & 0,003 & 7,39 & 1,97 & 0,004 & 0,004 & - & 0,04 & 0,076 & 0,128 \\
\hline $07 / 11 / 16$ & - & - & - & - & - & 0,004 & - & 0,04 & - & 0,291 \\
\hline $05 / 12 / 16$ & - & - & - & - & - & 0,004 & - & 0,04 & - & 0,264 \\
\hline $10 / 01 / 17$ & 0,002 & 0,002 & 4,11 & 2,07 & 0,004 & 0,004 & 0,04 & 0,04 & 0,148 & 0,084 \\
\hline LIMITE & \multicolumn{2}{|c|}{0,005} & \multicolumn{2}{|c|}{250} & \multicolumn{2}{|c|}{0,009} & \multicolumn{2}{|c|}{0,05} & \multicolumn{2}{|c|}{0,300} \\
\hline
\end{tabular}

Fonte: Dados: IGAM (2017). Elaboração do autor. . Valores acima dos limites estabelecidos pela Resolução CONAMA no $357 / 2005$ (negrito)

Verificamos na Tabela 3, que os níveis de fósforo apresentaram alterações nos valores dos dois pontos analisados, antes do rompimento da barragem de rejeitos. No ponto RD009, fora da rota de movimento dos rejeitos, foram constatadas três amostras fora dos padrões de um total de 12 amostras, enquanto no ponto RD071, apenas uma amostra em doze estava fora do padrão. Neste mesmo ponto de coleta após a passagem dos rejeitos, não foram mais identificados valores de fósforo elevados, enquanto que, no ponto RD009, sem contato com o rejeito, foram identificadas duas amostras fora do padrão de um total de cinco realizadas entre 11/01/2016 e 10/01/2017. De acordo com a ANA (2017) o fósforo é um importante nutriente para os processos biológicos. Pode levar ao processo de eutrofização dos rios quando em excesso nas águas, afetando a utilização normal e desejável da água devido ao acúmulo de matéria orgânica em decomposição. O fósforo é um indicativo de contaminação orgânica por esgotos domésticos ou de atividade pecuária, assim como de atividade agrícola, considerando a adubação com fertilizantes fosfatados. Existem indícios de que estas atividades são mais intensas na microbacia de captação referente ao ponto RD009, fora da rota dos rejeitos da barragem.

O manganês é um dos elementos identificados nos rejeitos da mineração da região de Mariana em quantidades relativamente elevadas. Observa-se que no ponto RD009, 
antes do rompimento da barragem, todas as coletas de agua realizadas apresentaram valores acima do preconizado pela resolução CONAMA, no total de cinco amostras, enquanto no ponto RD071, rota da barragem rompida, de 12 amostras coletadas foram encontrados valores elevados deste elemento em cinco delas. Após o rompimento da barragem, as amostras de água do ponto RD009, mesmo fora da rota dos rejeitos, manteve os valores elevados de manganês. No ponto de coleta RD071 verifica-se que ocorreu um agravamento da situação, com sete amostras de água coletadas apresentando valores elevados de manganês de um total de oito coletas. Provavelmente, alguma fonte de manganês que contaminava a água do rio do Carmo antes do rompimento da barragem, continuou após rompimento, potencializado pelos rejeitos da barragem que se rompeu. Em relação aos demais elementos descritos na Tabela 3, não foi observado qualquer situação amostral com valores acima do preconizado pela resolução CONAMA nos pontos analisados, antes e depois do rompimento.

O manganês apresenta toxidade baixa. Há poucos relatos de intoxicação por manganês via oral. Porém há estudos que relatam que crianças expostas a elevados níveis iguais ou superiores a $0,5 \mathrm{mg} / \mathrm{L}$ em água potável apresentaram déficits intelectuais. A forma mais comum de intoxicação é por via inalatória, em minas, siderúrgicas e algumas indústrias químicas. Há estudos que relataram distúrbios psiquiátricos graves em mineradores chilenos intoxicados por este metal (GIAIA, 2016).

$\mathrm{Na}$ Tabela 4, observa-se que para os elementos analisados não foi constatada qualquer alteração na qualidade da água nos pontos observados, antes e após o rompimento da barragem, com exceção do elemento zinco. O parâmetro zinco total possui apenas um valor que não atende a legislação, localizada no RD071 datado em outubro de 2016, apresentando não ser uma contaminação recorrente no Rio do Carmo e sugerindo ausência de relação com a descarga de rejeitos no rio. Cabe ressaltar que o consumo de 15-20mg de zinco é necessário na dieta alimentar diária para homens adultos, porém é tóxico quando ingerido em concentrações acima de $500 \mathrm{mg}$ de sulfato de zinco, e pode provocar náusea, febre, vômitos e perturbações gastrointestinais. (HADDAD, 2007).

Alguns autores descrevem os níveis destes elementos nos rejeitos de mineração da região como muito reduzidos e, desta forma, não comprometendo a qualidade da água na região. Outras fontes poderiam contribuir para a elevação destes valores nas fontes hídricas como a indústria, o esgoto ou a agropecuária, mas isto não ocorreu. 
Tabela 03- Parâmetros inorgânicos (fósforo, manganês, níquel, totais e nitrato, $\mathrm{mg} / \mathrm{L}$ ) antes do rompimento da barragem em Mariana (preto) e após o rompimento (vermelho) em pontos do Rio do Carmo sem interferência dos rejeitos da mineração (RD009) e com interferência (RD071)

\begin{tabular}{|c|c|c|c|c|c|c|c|c|c|c|}
\hline \multirow{2}{*}{ Data } & \multicolumn{2}{|c|}{ Fósforo Total } & \multicolumn{2}{|c|}{ Manganês Total } & \multicolumn{2}{|c|}{ Mercúrio Total } & \multicolumn{2}{|c|}{ Níquel Total } & \multicolumn{2}{|c|}{ Nitrato } \\
\hline & RD009 & RD00071 & RD009 & RD0071 & $\begin{array}{c}\text { RDOC } \\
9\end{array}$ & RD0071 & RD009 & RD0071 & RD009 & RD0071 \\
\hline $15 / 01 / 13$ & 0,05 & 0,05 & 1,390 & 0,622 & 0,2 & 0,2 & 0,005 & 0,008 & 0,9 & 0,30 \\
\hline $09 / 04 / 13$ & 0,22 & 0,19 & - & 1,558 & 0,2 & 0,2 & - & 0,008 & 1,41 & 0,96 \\
\hline $09 / 07 / 13$ & 0,10 & 0,02 & 0,750 & 0,043 & 0,2 & 0,2 & 0,004 & 0,004 & 1,70 & 0,57 \\
\hline $01 / 10 / 13$ & 0,10 & 0,02 & - & 0,171 & 0,2 & 0,2 & - & 0,004 & 1,22 & 0,54 \\
\hline $14 / 01 / 14$ & 0,08 & 0,03 & 0,910 & 0,297 & 0,2 & 0,2 & 0,004 & 0,004 & 1,63 & 0,59 \\
\hline $01 / 04 / 14$ & 0,06 & 0,02 & - & 0,057 & 0,2 & 0,2 & - & 0,004 & 1,35 & 0,58 \\
\hline $08 / 07 / 14$ & 0,09 & 0,02 & 1,300 & 0,035 & 0,2 & 0,2 & 0,004 & 0,004 & 1,74 & 0,58 \\
\hline $01 / 10 / 14$ & 0,14 & 0,02 & - & 0,063 & 0,2 & 0,2 & - & 0,004 & 2,00 & 0,58 \\
\hline 13/01/15 & 0,14 & 0,09 & 0,610 & 0,044 & 0,2 & 0,2 & 0,004 & 0,004 & 2,93 & 0,62 \\
\hline 07/04/15 & 0,06 & 0,06 & - & 0,206 & 0,2 & 0,2 & - & 0,004 & 0,77 & 0,84 \\
\hline 07/07/15 & 0,19 & 0,06 & 0,560 & 0,035 & 0,2 & 0,2 & 0,004 & 0,004 & 1,63 & 0,53 \\
\hline 06/10/15 & 0,14 & 0,04 & - & 0,067 & 0,2 & 0,2 & - & 0,004 & 1,52 & 0,36 \\
\hline $11 / 01 / 16$ & - & - & 1,610 & 1,375 & 0,2 & 0,2 & 0,004 & 0,004 & 1,46 & 0,54 \\
\hline $12 / 01 / 16$ & 0,38 & 0,04 & - & 0,309 & 0,2 & 0,2 & - & 0,004 & 1,33 & 0,58 \\
\hline 05/04/16 & 0,08 & 0,07 & - & - & - & - & - & - & - & - \\
\hline 05/07/16 & 0,14 & 0,02 & 0,880 & 0,061 & 0,2 & 0,2 & 0,004 & 0,004 & 1,96 & 0,48 \\
\hline $30 / 09 / 16$ & - & - & - & 0,318 & - & 0,2 & - & 0,004 & - & - \\
\hline 18/10/16 & 0,09 & 0,04 & 0,019 & 0,199 & 0,2 & 0,2 & - & 0,004 & 2,25 & 0,66 \\
\hline 07/11/16 & - & - & - & 0,159 & - & 0,2 & - & 0,004 & - & - \\
\hline 05/12/16 & - & - & - & 1,143 & - & 0,2 & - & 0,005 & - & - \\
\hline $10 / 01 / 17$ & 0,07 & 0,04 & 1,030 & 0,230 & 0,2 & 0,2 & 0,004 & 0,004 & 1,34 & 0,63 \\
\hline LIMITES & \multicolumn{2}{|c|}{0,10} & \multicolumn{2}{|c|}{0,100} & \multicolumn{2}{|r|}{0,2} & \multicolumn{2}{|c|}{0,025} & \multicolumn{2}{|c|}{10,00} \\
\hline
\end{tabular}

Fonte: Dados: IGAM (2017). Elaboração do autor. . Valores acima dos limites estabelecidos pela Resolução CONAMA nº 357/2005 (negrito)

Tabela 04- Parâmetros inorgânicos (Nitrito, nitrogênio amoniacal total (NAT), sulfato total, sulfeto e zinco total, mg/L) antes do rompimento da barragem em Mariana (preto) e após o rompimento (vermelho) em pontos do Rio do Carmo sem interferência dos rejeitos da mineração (RD009) e com interferência (RD071)

\begin{tabular}{|c|c|c|c|c|c|c|c|c|c|c|}
\hline \multirow{3}{*}{ Data } & \multirow{2}{*}{\multicolumn{2}{|c|}{ Nitrito }} & \multirow{2}{*}{\multicolumn{2}{|c|}{ NAT }} & \multirow{2}{*}{\multicolumn{2}{|c|}{$\begin{array}{l}\text { Sulfato Total } \\
\text { RD00 }\end{array}$}} & \multicolumn{2}{|c|}{ Sulfeto } & \multicolumn{2}{|c|}{ Zinco Total } \\
\hline & & & & & & & \multirow{2}{*}{ RD009 } & \multirow{2}{*}{ RD0071 } & \multirow{2}{*}{ RD009 } & \multirow{2}{*}{ RD0071 } \\
\hline & RD009 & RD0071 & RD009 & RD0071 & 9 & RD0071 & & & & \\
\hline $15 / 01 / 13$ & 0,098 & 0,009 & 0,57 & 0,10 & 6,9 & 5,0 & 0,01 & 0,01 & 0,02 & 0,020 \\
\hline $09 / 04 / 13$ & - & 0,012 & 0,18 & 0,11 & - & - & - & 0,05 & - & 0,056 \\
\hline $09 / 07 / 13$ & 0,105 & 0,005 & 0,19 & 0,11 & 7,0 & 5,0 & 0,01 & 0,02 & 0,02 & 0,020 \\
\hline $01 / 10 / 13$ & - & 0,010 & 0,59 & 0,17 & - & - & - & 0,01 & - & 0,025 \\
\hline $14 / 01 / 14$ & 0,188 & 0,009 & 0,29 & 0,16 & 8,8 & 5,0 & 0,01 & 0,02 & 0,024 & 0,020 \\
\hline $01 / 04 / 14$ & - & 0,008 & 0,10 & 0,10 & - & - & - & 0,04 & - & 0,020 \\
\hline $08 / 07 / 14$ & 0,226 & 0,005 & 0,68 & 0,10 & 5,8 & 5,0 & 0,01 & 0,01 & 0,025 & 0,020 \\
\hline $01 / 10 / 14$ & - & 0,007 & 0,10 & 0,10 & - & - & - & 0,01 & - & 0,020 \\
\hline $13 / 01 / 15$ & 0,160 & 0,006 & 0,13 & 0,10 & 8,5 & 10,3 & 0,01 & 0,01 & 0,020 & 0,020 \\
\hline $07 / 04 / 15$ & - & 0,011 & 0,10 & 0,10 & - & - & - & 0,01 & - & 0,020 \\
\hline $07 / 07 / 15$ & 0,154 & 0,005 & 0,11 & 0,10 & 15,5 & 5,9 & 0,01 & 0,01 & 0,020 & 0,020 \\
\hline $06 / 10 / 15$ & - & 0,003 & 0,10 & 0,10 & - & - & - & 0,01 & - & 0,027 \\
\hline $11 / 01 / 16$ & 0,221 & 0,012 & 0,10 & 0,10 & 53,1 & 7,9 & 0,01 & 0,01 & 0,042 & 0,028 \\
\hline $12 / 01 / 16$ & - & 0,019 & 0,13 & 0,14 & - & - & - & 0,01 & - & 0,029 \\
\hline $05 / 04 / 16$ & - & - & - & - & - & - & - & 0,01 & - & - \\
\hline $05 / 07 / 16$ & 0,161 & 0,002 & 0,10 & 0,10 & 35,1 & 5,0 & 0,01 & 0,01 & 0,020 & 0,025 \\
\hline $30 / 09 / 16$ & - & - & 0,14 & - & - & - & - & - & - & 0,020 \\
\hline $18 / 10 / 16$ & - & 0,004 & - & 0,10 & - & - & - & 0,01 & - & 0,439 \\
\hline $07 / 11 / 16$ & - & - & - & - & - & - & - & - & - & 0,020 \\
\hline $05 / 12 / 16$ & - & - & 0,21 & - & - & - & - & - & - & 0,020 \\
\hline $10 / 01 / 17$ & 0,122 & 0,003 & & & 34,5 & 5,7 & 0,01 & 0,01 & - & - \\
\hline LIMITES & \multicolumn{2}{|c|}{1,000} & \multicolumn{2}{|c|}{$\begin{array}{l}3,7 \mathrm{mg} / \mathrm{L} \mathrm{N} \text {, para } \mathrm{pH} \leq 7,52,0 \\
\mathrm{mg} / \mathrm{L} \mathrm{N} \text {, para } 7,5<\mathrm{pH} \leq 8,0 \\
1,0 \mathrm{mg} / \mathrm{L} \mathrm{N} \text {, para } 8,0<\mathrm{pH} \leq \\
8,50,5 \mathrm{mg} / \mathrm{L} \mathrm{N}, \text { para } \mathrm{pH}>8,5\end{array}$} & \multicolumn{2}{|c|}{250,0} & \multicolumn{2}{|c|}{0,002} & \multicolumn{2}{|c|}{0,180} \\
\hline
\end{tabular}

Fonte: Dados: IGAM (2017). Elaboração do autor. . Valores acima dos limites estabelecidos pela Resolução CONAMA ํㅜ $357 / 2005$ (negrito)

A presença de alguns metais pesados no rio do Carmo tem muita influência também da secular atividade garimpeira. Isso ocorre devido a disponibilização de grande parte do 
material do garimpo nos rios pelos rejeitos das minas de ouro de Ouro Preto e Mariana durante três séculos de exploração mineral na região (COSTA et al., 2006). O rio Gualaxo do Norte recebe os efluentes das minas de ferro de Timbopeba, Capanema, Samarco e Samitri e de garimpos. A exploração do minério de ferro e do ouro, bem como o processo de beneficiamento, produz derivados do processo e através desses é capaz de disponibilizar diferentes contaminantes ao rio (RHODES, 2010).

Costa et al (2010) observou concentrações de arsênio mais elevadas em sedimentos da bacia do ribeirão do Carmo, em relação a bacia do rio Gualaxo do Norte. Tal observação pode ter forte influência da mineração de ouro e no enriquecimento de arsênio na região.

Nas Tabela 05 e 06 são apresentados os resultados das análises do monitoramento para os demais parâmetros avaliados, os quais, clorofila, cor verdadeira, demanda bioquímica de oxigênio (DBO), fenóis totais, óleos e graxas, oxigênio dissolvido (OD), pH in loco, sólidos dissolvidos totais (SDT) e turbidez. Observa-se que todos os parâmetros atendem aos requisitos normativos, com exceção da turbidez.

Valores acima dos estabelecidos para o parâmetro turbidez (Tabela 6) são encontrados nos pontos de monitoramento em estudo desde 2013, porém é possível observar concentrações extremamente elevadas no dia 09 de abril de 2013, apresentando 744 UNT, no dia 12 de janeiro de 2016 apresentando 3365 UNT e no dia 05 de dezembro de 2016 apresentando valores de 5228 UNT, mais de um ano após o rompimento da barragem de Fundão, valores no ponto RD071. O máximo permitido pelas legislações é 40 UNT, demonstrando o quão elevado são esses resultados. O resultado 3365 de turbidez ocorreu logo após a data do rompimento da barragem de Fundão e na RD009 na mesma data o valor foi de 45,1 UNT, pouco acima do estabelecido, indicando forte indícios de relação com o acidente ocorrido e capaz de alterar as características organolépticas (cor, sabor, aparência e odor) da água e levar a rejeição para fins de potabilidade. O aumento da turbidez também se relaciona indiretamente com a fotossíntese. Essa relação permite a alteração da cadeia trófica aquática, podendo causar a mortandade de peixes devido a influência nas comunidades biológicas na água (HADDAD, 2007). Outro possível efeito da turbidez elevada é mascarar a presença de possíveis organismos patogênicos bem como estimular o crescimento de bactérias devido a nutrientes que são absorvidos nas partículas (WHO, 2011). 
O aumento da turbidez exige das estações de tratamento de água a utilização de uma quantidade maior de produtos químicos, aumentando os custos do tratamento, por dificultar o processo de clareamento da água. A turbidez afeta também a preservação dos organismos aquáticos, o uso industrial e as atividades de recreação (ANA, 2017).

Tabela 05- Parâmetros Clorofila a (ug/L), cor verdadeira, DBO ( $\left.\mathrm{mgO}_{2} / \mathrm{L}\right)$, fenóis totais (mg/L), óleos e graxas $(\mathrm{mg} / \mathrm{L})$ antes do rompimento da barragem em Mariana (preto) e após o rompimento (vermelho) em pontos do Rio do Carmo sem interferência dos rejeitos da mineração (RD009) e com interferência (RD071)

\begin{tabular}{|c|c|c|c|c|c|c|c|c|c|c|}
\hline \multirow[b]{2}{*}{ Data } & \multicolumn{2}{|c|}{ Clorofila a } & \multicolumn{2}{|c|}{ Cor Verdadeira } & \multicolumn{2}{|c|}{ DBO } & \multicolumn{2}{|c|}{ Fenóis Totais } & \multicolumn{2}{|c|}{ Óleos e Graxas } \\
\hline & $\begin{array}{l}\mathrm{RD} 00 \\
9\end{array}$ & RD071 & $\begin{array}{l}\text { RD00 } \\
9\end{array}$ & RD071 & RD009 & RD071 & RD009 & $\begin{array}{c}\mathrm{RD} 07 \\
1\end{array}$ & RD009 & RD071 \\
\hline $15 / 01 / 13$ & 0,53 & 1,07 & 10 & 29 & 2,0 & 2,0 & 0,002 & 0,002 & 15 & 15 \\
\hline $09 / 04 / 13$ & 6,67 & 12,68 & - & - & 2,7 & 2,0 & 0,002 & 0,002 & - & - \\
\hline 09/07/13 & 1,33 & 0,01 & 10 & 10 & 2,0 & 2,0 & 0,002 & 0,002 & 15 & 15 \\
\hline $01 / 10 / 13$ & 0,53 & 3,20 & - & - & 4,8 & 2,0 & 0,002 & 0,002 & - & - \\
\hline $14 / 01 / 14$ & 1,20 & 0,40 & 10 & 24 & 2,0 & 2,0 & 0,002 & 0,002 & 15 & 15 \\
\hline $01 / 04 / 14$ & 0,53 & 0,80 & - & - & 2,0 & 2,0 & 0,002 & 0,002 & - & - \\
\hline $08 / 07 / 14$ & 1,33 & 0,27 & 10 & 10 & 4,3 & 2,0 & 0,002 & 0,002 & 15 & 15 \\
\hline $01 / 10 / 14$ & 18,02 & 0,01 & - & - & 3,7 & 2,0 & 0,002 & 0,002 & 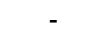 & - \\
\hline 13/01/15 & 0,67 & 1,73 & 12 & 14 & 2,0 & 2,0 & 0,002 & 0,002 & 15 & 15 \\
\hline 07/04/15 & 0,13 & 0,27 & - & - & 2,0 & 2,0 & 0,002 & 0,002 & - & - \\
\hline 07/07/15 & 2,80 & 2,40 & 10 & 10 & 2,1 & 2,0 & 0,002 & 0,002 & 15 & 15 \\
\hline $06 / 10 / 15$ & 4,69 & 1,33 & - & - & 2,0 & 2,0 & 0,002 & 0,002 & - & - \\
\hline $11 / 01 / 16$ & 2,40 & - & - & - & - & - & - & - & 15 & 15 \\
\hline $12 / 01 / 16$ & 2,40 & 0,01 & 17 & 33 & 3,7 & 2,0 & 0,002 & 0,002 & - & - \\
\hline $05 / 04 / 16$ & 0,40 & 0,40 & - & - & 2,0 & 2,0 & 0,002 & 0,002 & - & - \\
\hline 05/07/16 & 3,87 & 2,14 & 11 & 15 & 2,2 & 2,0 & 0,002 & 0,002 & 15 & 15 \\
\hline $30 / 09 / 16$ & - & - & - & - & - & - & - & - & - & - \\
\hline $18 / 10 / 16$ & 0,80 & 1,33 & - & - & 2,0 & 2,0 & 0,002 & 0,002 & - & - \\
\hline 07/11/16 & - & - & - & - & - & - & - & - & - & - \\
\hline $05 / 12 / 16$ & - & - & - & - & - & - & - & - & - & - \\
\hline $10 / 01 / 17$ & 2,31 & 1,17 & 10 & 31 & 2,0 & 2,0 & 0,002 & 0,002 & - & - \\
\hline LIMITE & \multicolumn{2}{|c|}{30,00} & \multicolumn{2}{|c|}{75} & \multicolumn{2}{|c|}{5,0} & \multicolumn{2}{|c|}{0,003} & \multicolumn{2}{|c|}{$\begin{array}{l}\text { Virtualmente } \\
\text { Ausentes }\end{array}$} \\
\hline
\end{tabular}

Fonte: Dados: IGAM (2017). Elaboração do autor. . Valores acima dos limites estabelecidos pela Resolução CONAMA no $357 / 2005$ (negrito) 
Tabela 06 - Parâmetros Oxigênio dissolvido, sólidos dissolvidos totais(SDT), turbidez (UNT) antes do rompimento da barragem em Mariana (preto) e após o rompimento (vermelho) em pontos do Rio do Carmo sem interferência dos rejeitos da mineração (RD009) e com interferência (RD071)

\begin{tabular}{|c|c|c|c|c|c|c|c|c|}
\hline \multirow[b]{2}{*}{ Data } & \multicolumn{2}{|c|}{$\begin{array}{c}\text { Oxigênio } \\
\text { Dissolvido }\end{array}$} & \multicolumn{2}{|c|}{$\mathrm{pH}$} & \multicolumn{2}{|c|}{ SDT } & \multicolumn{2}{|c|}{ Turbidez } \\
\hline & RD009 & RD071 & RD009 & RD071 & RD009 & RD071 & RD009 & RD071 \\
\hline $15 / 01 / 13$ & 6,7 & 7,6 & 6,9 & 6,8 & 61 & 36 & 46,20 & 72,90 \\
\hline $09 / 04 / 13$ & 7,1 & 7,9 & 6,5 & 6,7 & 41 & 96 & 257,00 & 744,00 \\
\hline 09/07/13 & 8,0 & 9,1 & 6,9 & 6,8 & 62 & 36 & 4,59 & 5,23 \\
\hline $01 / 10 / 13$ & 6,6 & 8,2 & 7,0 & 6,9 & 66 & 54 & 23,60 & 41,70 \\
\hline $14 / 01 / 14$ & 6,9 & 7,6 & 7,2 & 6,9 & 56 & 28 & 7,60 & 29,50 \\
\hline $01 / 04 / 14$ & 7,2 & 8,3 & 7,2 & 7,3 & 78 & 48 & 7,25 & 6,87 \\
\hline $08 / 07 / 14$ & 7,2 & 8,2 & 6,9 & 6,7 & 85 & 48 & 43,30 & 2,73 \\
\hline $01 / 10 / 14$ & 7,5 & 7,6 & 7,4 & 7,3 & 151 & 53 & 5,30 & 4,21 \\
\hline $13 / 01 / 15$ & 6,5 & 7,7 & 7,3 & 7,6 & 111 & 45 & 3,30 & 3,56 \\
\hline $07 / 04 / 15$ & 6,7 & 7,8 & 6,8 & 6,4 & 83 & 46 & 6,78 & 15,80 \\
\hline $07 / 07 / 15$ & 8,7 & 9,2 & 7,5 & 7,8 & 33 & 43 & 2,10 & 1,85 \\
\hline $06 / 10 / 15$ & 7,8 & 8,3 & 6,6 & 6,5 & 124 & 51 & 11,00 & 1,80 \\
\hline $11 / 01 / 16$ & 6,4 & 7,5 & 7,1 & 6,9 & 132 & 112 & 45,10 & 3365,00 \\
\hline $12 / 01 / 16$ & 7,0 & 7,9 & 7 & 7,1 & 125 & 68 & 6,31 & \\
\hline $05 / 04 / 16$ & - & - & - & - & - & - & - & 135,00 \\
\hline 05/07/16 & 7,9 & 9,0 & 6,9 & 7,1 & 142 & 110 & 4,73 & 41,70 \\
\hline $30 / 09 / 16$ & - & 8,0 & - & 7,2 & - & 66 & - & 145,00 \\
\hline $18 / 10 / 16$ & 6,3 & 7,8 & 7,4 & 7,4 & 248 & 116 & 7,97 & 93,70 \\
\hline $07 / 11 / 16$ & - & 8,0 & - & 7,2 & - & 100 & - & 58,70 \\
\hline $05 / 12 / 16$ & - & 7,9 & - & 6,2 & - & 142 & - & 5228,00 \\
\hline \multirow{2}{*}{$\begin{array}{r}10 / 01 / 17 \\
\text { LIMITE }\end{array}$} & 6,5 & 7,4 & 6,7 & 6,4 & 116 & 80 & 39,10 & 161,00 \\
\hline & \multicolumn{2}{|c|}{$\begin{array}{c}\text { Não inferior a } \\
6,0 \mathrm{mg} / \mathrm{L}\end{array}$} & \multicolumn{2}{|c|}{6,0 a 9,0} & \multicolumn{2}{|c|}{500} & \multicolumn{2}{|c|}{40,00 UNT } \\
\hline
\end{tabular}

Fonte: Dados: IGAM (2017). Elaboração do autor. . Valores acima dos limites estabelecidos pela Resolução CONAMA no $357 / 2005$ (negrito)

\section{CONCLUSÃO}

A degradação ambiental e suas consequências para o planeta terra e a saúde pública é uma preocupação e que se torna mais evidente a cada dia. $\mathrm{Na}$ análise dos parâmetros inorgânicos dos pontos de monitoramento verificou-se problemas mais frequentes com os elementos fósforo, manganês, alumínio e arsênio. Não foi possível relacionar os valores ou constatar indícios de que as concentrações dos elementos que não atendem legislação estejam relacionadas com o acidente do rompimento da barragem de Fundão em Mariana-MG. Por outro lado, foi possível constatar que não há totalidade de atendimento aos limites de concentração previstos na legislação, indicando poluição hídrica e necessidade de maiores pesquisas na busca das fontes.

Dentre os demais parâmetros de qualidade de água analisados, constatou-se que apenas turbidez possui valor que não atende aos requisitos normativos.

O estudo sugere que as inconformidades nos parâmetros encontradas no rio do Carmo não estão necessariamente relacionadas com o rompimento da barragem, com exceção da turbidez, indicando que o rio já possuía parâmetros fora do padrão antes do rompimento, bem como à montante do deságue do rio Gualaxo do Norte. 
Este estudo demonstrou a necessidade da continuidade do monitoramento e das pesquisas sobre as fontes com atuação sobre as mesmas e implantação e medidas mitigadoras.

\section{REFERÊNCIAS}

AGÊNCIA NACIONAL DE ÁGUAS (ANA). Conjuntura dos recursos hídricos no Brasil: informe 2015. Encarte especial sobre a bacia do Rio Doce- rompimento da barragem de Mariana, Superintendência de Planejamento de Recursos Hídricos - SPR Brasília - DF, 2016

AGÊNCIA NACIONAL DE ÁGUAS (ANA). Indicadores de qualidade: índice de qualidade das águas (IQA).2017. Disponível em: <http://portalpnqa.ana.gov.br/indicadores-indiceaguas.aspx\#_ftn8>. Acesso em: 20 dez. 2017.

BARBOSA, S. E. da S.; JÚNIOR, A. R. B.; SILVA, G. Q.; CAMPOS, E. N. B.; RODRIGUES, V. C. Geração de modelos de regionalização de vazões máximas, médias de longo período e mínimas de sete dias para a bacia do rio do Carmo, Minas Gerais. Engenharia Sanitária e Ambiental, v. 10, n.1, p.64-71, 2005.

CONSELHO NACIONAL DO MEIO AMBIENTE (CONAMA). Resolução no 357. Diário Oficial da União de 17 de março de 2005.

CAVACO, M. H. educação ambiental para o desenvolvimento: testemunhos e notícias. Cadernos de Inovação Educacional. Lisboa: Escolar Editora. 1992, 148p.

COSTA A.T., NALINI-JR H.A., CASTRO P.T.A., FRIESE K. Sediment contamination in floodplains and alluvial terraces as an historical record of gold exploitation in the Carmo River basin, Southeast Quadrilátero Ferrífero, Minas Gerais, Brazil. Acta Hydrochimica et Hydrobiologica, v.34, p.245256, 2006.

COSTA A.T., NALINI-JR H.A., CASTRO P.T.A., TATUMI S.H. Análise estratigráfica e distribuição do arsênio em depósitos sedimentares quaternátios da porção sudeste do Quadrilátero Ferrífero, bacia do Ribeirão do Carmo, MG. Revista Escola de Minas, v. 63. n.4, p.703-714, 2010.

HADDAD, E. A. Influência antrôpica na qualidade da água da bacia hidrográfica do Rio São Miguel, Carste do Alto São Francisco, Minas Gerais. 2007. 156 f. Dissertação (Mestrado) - Curso de Programa de Pós Graduação em Geografia, Departamento de Geografia, Universidade Federal de Minas Gerais, Belo Horizonte, 2007.

INSTITUTO DE GESTÃO DAS ÁGUAS DE MINAS GERAIS (IGAM). Plano integrado de recursos hídricos da bacia do rio doce e dos planos de ações de recursos hídricos para as unidades de planejamento e gestão de recursos hídricos no âmbito da bacia do Rio Doce. Plano de Ação de Recursos Hídricos da Unidade de Planejamento e Gestão dos Recursos Hídricos Piranga. Maio 2010.

INSTITUTO DE GESTÃO DAS ÁGUAS DE MINAS GERAIS (IGAM). Monitoramento da qualidade das águas superficiais em minas gerais - relatório trimestral- 3 trimestre 2013, SEMAD - 
Secretaria de Estado de Meio Ambiente e Desenvolvimento Sustentável, Governo do estado de Minas Gerais, 2013.

INSTITUTO DE GESTÃO DAS ÁGUAS DE MINAS GERAIS (IGAM). Monitoramento da qualidade das águas superficiais do rio Doce no estado de Minas Gerais: acompanhamento da qualidade das Águas do Rio Doce Após o Rompimento da Barragem da Samarco no distrito de Bento Rodrigues - Mariana/MG. Belo Horizonte, 2016. 75 p.

INSTITUTO DE GESTÃO DAS ÁGUAS DE MINAS GERAIS (IGAM). - Portal INFOHIDRO Sistema Estadual de Informações sobre Recursos Hídricos de Minas Gerais. 2017. Disponível em: < http://portalinfohidro.igam.mg.gov.br/>. Acesso em: 22 nov. 2017.

LIMA, V. F.; MERÇON, F. Metais Pesados no Ensino de Química. Química Nova na Escola, São Paulo, v. 33, n. 4, p.199-205, nov. 2011. Disponível em: <http://qnesc.sbq.org.br/online/qnesc33_4/199-CCD-7510.pdf>. Acesso em: 11 dez. 2017.

MACHADO, C. S.; ALVES, R. I. S.; FREGONESI, B. M.; TONANI, K. A. A.; DE MARTINIS, B. S. ; SIERRA, J. ; NADAL, M. ; DOMINGO, J. L.; SEGURA-MUÑOZ, S. I. Chemical contamination of water and sediments in the Pardo River, São Paulo, Brazil. Procedia Engineering, v.162, p.230237, 2016.

PEREIRA, R.S. Identificação e caracterização das fontes de poluição em sistemas hídricos. Revista Eletrônica de Recursos Hídricos, v.1, n.1, p. 20-36, 2004.

POLÍTICA NACIONAL DE RECURSOS HÍDRICOS (PNRH). Lei n 9.433. Presidência da República Federativa do Brasil. Brasília, de 8 de janeiro de 1997.

RHODES, V. da P. Distribuição de mercúrio e arsênio nos sedimentos de área afetada por garimpo de ouro - rio Gualaxo do Norte, Mariana-MG. 2010. 92f. Dissertação (Mestrado) Universidade Federal de Ouro Preto, Ouro Preto, 2010.

ROSA, A. H.; FRACETO, L. F.; MOSCHINI-CARLOS, V. Meio Ambiente e sustentabilidade. Porto Alegre: Bookman Companhia Editora Ltda, 2012. 412 p.

VICTORINO, C. J. A. Planeta água morrendo de sede: uma visão analítica na metodologia do uso e abuso dos recursos hídricos. Porto Alegre: EDIPUCRS. 2007, 231 p.

WORLD HEALTH ORGANIZATION (WHO). Lead in Drinking-water. Background document for development of WHO Guidelines for Drinking-water Quality. World Health Organization, 4nd. Ed., Geneva. 2011, 564p.

YABE, M. J. S.; OLIVEIRA, E. de. Metais pesados em águas superficiais como estratégia de caracterização de bacias hidrográficas. Química Nova. v.5, n.21, p.551-556, 1998. 